\title{
Strategies for preventing and screening for coronary heart disease
}

\author{
M F OLIVER \\ From the Cardiovascular Research Unit, University of Edinburgh, Edinburgh
}

"Strategy is simple, but not easy"-Clausewitz.

Intervention in the whole population in order to reduce the risk of coronary heart disease through altering lifestyles and specific intervention in individuals with identified high risk are complementary and not opposing strategies. Yet they are valid alternatives in that they present society with different choices and uses of its limited resources. To community health educators-and evidently to epidemiologists-mass intervention is to be preferred. To many physicians and cardiologists, the high risk strategy is likely to be more attractive. In the best of all possible worlds we should be able to afford both policies if they truly benefit society.

But, so far, the results of investment in mass intervention trials have not been impressive-with a very considerable outlay and little or no yield (see later). It is argued that such trials are unnecessary because the cost of changing lifestyle on a mass population basis is really negligible and it is not expensive to promote campaigns to tell people to stop smoking, change their diet, or take more exercise: such advice is bound to do more good than harm, it is said.

Yet improved health is not the only need of the population. Simply to argue that cigarette smoking should be eliminated without taking into account the repercussions on economy and employment is a narrow medical view. Similarly, to recommend changes in farming policy and farming economy leading to reductions in dairy farming by a quarter or a third needs much more consideration than it usually gets from the health education enthusiasts. Reduction in milk production and the requirement for less carcass fat imply a substantial reduction in animal food, of which barley is the major component.' Land will be released for other purposes but perhaps more than is needed for fruit and vegetables. The case for making large changes in agricultural strategy, with all its

Requests for reprints to Professor M F Oliver, Cardiovascular Research Unit, Hugh Robson Building, George Square, Edinburgh EH8 9XF. repercussions on cereal, fruit, vegetable, and edible oil production and also on the welfare of those working in these industries, should surely be based on clear evidence that health will improve consequently. This evidence is not yet forthcoming.

\section{Mass intervention for moderate risk}

Une of the underlying problems with regard to intervention to prevent coronary heart disease is the very low specificity with which we are able to identify individuals at risk. The strength of the concept relating risk factors to coronary heart disease lies in their relative or proportional risk and not in their absolute risk or predictive power. While it is undeniable that those with serum cholesterol concentrations or blood pressure in the top $10 \%$ or $20 \%$ of the population distribution have a higher risk than those with lower levels, ${ }^{2-4}$ the actual risk is low. Thus two thirds of healthy adult men, aged $40-55$ years, found to have the highest risk (above the eightieth centile) as a result of raised cholesterol concentrations (excluding familial hypercholesterolaemia) and blood pressure can be estimated to remain well over the subsequent 25 years. ${ }^{2}$ Hopefully, this low specificity will improve when some of the emerging measurements of thrombogenic risk have been evaluated as predictors.

It should not be a surprise, therefore, or particularly controversial, that intervention to control raised cholesterol concentrations and raised blood pressure in healthy adult men who are at moderate risk, even when conducted simultaneously and in conjunction with reduction of cigarette smoking, has not been successful on a mass basis. ${ }^{5-7}$ What lessons are there to learn? One argument, given in defence by enthusiasts, is that too little was done too late. We will only know if this is true when more has been done earlier. But it is impracticable to do much more: for example, attempts were made to make a profound change in the consumption of dietary fat, along the lines recommended by the World Health Organisa- 
tion, ${ }^{8}$ in the Multiple Risk Factor Intervention Trial ${ }^{5}$ but it is quite clear from the disappointing reduction in plasma cholesterol concentrations (let alone the lack of change in the incidence of coronary heart disease) that compliance was bad-in spite of advice received from teams of physicians, dietitians, sociologists, and psychologists. To do more with regard to the control of blood pressure means the life time use of potent drugs with their attendant side effects. More might be done, however, with regard to cigarette smoking, although the chances of eliminating this are negligible. Another common argument from those committed to mass intervention is that five to 10 years is too shork a period to appraise results of trials because coronary atherosclerosis takes many years to develop and an equal period may be necessary to prove the effectiveness of changes in lifestyle on coronary heart disease. May be, but coronary atherosclerosis and coronary heart disease do not equate, and even successful demonstration of regression of coronary atherosclerosis does not necessarily mean less coronary heart disease. There are no large randomised multiple risk factor intervention trials against coronary heart disease proceeding into a second decade of testing, and so how shall we know? Evidently, we will have to rely on being able to interpret national trends in coronary heart disease mortality rates in terms of prevention policies, but there are so many confounding influences that this does not look promising.

\section{Selective intervention for high risk}

The scientific evidence for intervention in those at high risk for coronary heart disease on account of hypercholesterolaemia is much better than for those at moderate risk. But it is still unsure because the numbers in the two main trials-the Uslo trial' ${ }^{y}$ and the Lipid Research Clinic Coronary Primary Prevention Trial, ${ }^{10}$ in which very high (above the ninetieth percentile) serum cholesterol concentrations were reduced-are too small and the margins of difference in coronary heart disease rates too narrow for it to be certain that whether and how big a proportion of people will benefit from treatment. In the Uslo Heart Study, comprising 1232 men aged 40-49, there was a significant reduction $(p<0.03)$ in the mixed end point of cardiac death and non-fatal myocardial infarction; but there was one case of sudden death in the control group with was "unexplained" and, were this case not to be due to coronary heart disease and were it to have been randomised to the intervention group, total coronary heart disease and total cardiovascular events would not have been significant at the $5 \%$ level." The Lipid Research Clinic Coronary Primary Prevention Trial showed a $19 \%$ fall, again in a mixed end point of coronary deaths and non-fatal myocardial infarction, at the $4 \%$ level of significance using a one tail test of significance. While the confidence limits of $+3 \%$ and $+32 \%$ (or $-0.1 \%$ and $+3.5 \%$ considering difference in risk (I) surrounding the main end points do not provide a sound enough basis to argue, as the authors have done, that these results provide the basis for lowering moderately raised cholesterol and low density lipoproteins in populations less highly at risk, the consistency between the results of these two trials is impressive. Additionally, there were in the Lipid Research Clinic Coronary Primary Prevention Trial non-significant reductions in the incidence of angina, abnormal exercise electrocardiogram tests, and referrals for coronary artery surgery. There was also a reduced rate of coronary heart disease incidence in three other trials of reducing high concentrations of serum cholesterol-the World Health Urganisation clofibrate trial ${ }^{12}$ and the Helsinki Mental Hospitals ${ }^{13}$ and Veterans Administration Studies, ${ }^{14}$ where diets with a high ratio of polyunsaturated to saturated fats were used.

The frailty of the case is added to by the fact that all these studies, including the dietary intervention trials, showed an increase in non-cardiovascular mortality, and in several of them the order of magnitude of this increase was comparable to that of the decrease in coronary heart disease. ${ }^{15}$ The increase in noncardiovascular mortality in the World Health Urganisation clofibrate trial has recently been shown to relate closely to the period of administration of the drug, ${ }^{16}$ and the increase in the incidence of oralgastrointestinal cancers which occurred in the Lipid Research Clinic Coronary Primary Prevention cholestyramine trial should not be dismissed as a random change in cancer prevalence, since a plausible explanation-a change in local $\mathrm{pH}$ due to the anion-exchange resin-may be advanced.

It has been argued ${ }^{17}$ that the results of all trialswhen lumped together, regardless of treatment and regardless of design, numbers, and mixed end points-show a mathematically convincing benefit for coronary heart disease. This is, to me, too simplistic biologically: but, even if it is a valid way to appraise the results of trials, the same approach shows an adverse effect for total and non-cardiovascular mortality which cannot easily be dismissed.

The basis for intervening in those at high risk on account of severe hypertension is that the incidence of strokes is less. Were this not so, it might be hard to make a strong case for lowering moderately raised blood pressure in order to reduce coronary heart disease for the evidence of benefit is unconvincing ${ }^{18}$ and may even, in those with pre-established signs of myocardial ischaemia, be harmful.

Nevertheless, I think that the case for intervention 
in individuals at very high risk for coronary heart disease is greater than the case for doing nothing and that we are justified in exposing them to the risks of drugs and inconvenience of stringent dietary changes. The American Heart Association has recently made appropriate recommendations for the management of such people. ${ }^{19}$ How may we best identify such individuals?

\section{To screen or not to screen}

Just as there are alternative conceptual approaches to intervention, there are alternatives for screening the population in order to identify people most at risk for coronary heart disease.

Screening is irrelevant to those who propound a policy of mass prevention since they wish to advise the whole population to change its lifestyle now. But for those who have doubts about the value and practicability of mass intervention and who endorse the high risk strategy the difficult question of how to screen the population simply, cheaply, effectively, and without producing anxiety must be addressed.

The alternatives are to screen the male adult population or to try to identify, mostly through clinical expertise, those at maximum risk (case finding). Uur individual and collective approach to these will depend to an extent on that part of medicine we represent-community care doctors or practising physicians and cardiologists.

The best policy would, at present, seem to be that we should aim at identifying those above the eightieth percentile of the distribution of serum cholesterol concentrations or blood pressure for that population for which it is planned to give advice (this is not necessarily the same numerical cut off point for all populations). There are three reasons for adopting this attitude. Une is that the relation of a given risk factor to the subsequent development of coronary heart disease is increasingly diluted by other known and unknown factors at lower deciles-in other words, specificity decreases. It is relatively good at high levels: recent figures for the Multiple Risk Factor Intervention Trial indicate that nearly $50 \%$ of cases of coronary heart disease "attributable" to high serum cholesterol concentrations occur in those with serum concentrations above the eightieth percentile, and $30 \%$ of cases occurred in those above the ninetieth percentile ${ }^{20}$; and the United Kingdom/World Health Organisation trial has also shown, taking four factors into account, that $32 \%$ of "attributable" cases occur in those above the eighty fifth percentile of risk. ${ }^{21} \mathrm{~A}$ second reason is that successful treatment of very high concentrations of serum cholesterol and of blood pressure require the use of drugs; and these should be acceptable ethically to physicians and cardiologists only when the risk of the disease is at least equal to the risk of giving drugs ${ }^{22}$ and to symptomless people when there is a really valid reason for making big chances in their lifestyle and committing them to drugs for the rest of their days. A third reason for a high cut off point is that the ratio of cost to benefit increases when intervention is attempted below the eightieth percentile owing to greater use and therefore costs of drugs in relation to a decreasing amount of ultimate benefit to be expected.

\section{Screening of all male adults}

Two considerations at least need to be thought through by those who advocate screening of the adult male population for subsequent implementation of high risk intervention against coronary heart disease. One is the practicability, costs, and advantages to society of universal screening and the other is possible disadvantages and the advice to be given to those for whom specific intervention is not really indicated. Incidentally, data concerning the relation of risk factors to coronary heart disease in women are far less well assembled than for men, and none of the clinical trials referred to above have included women: the probability is that the prevalence of risk factors is lower and that their significance is less and, until more formal evidence is available, a case for screening the adult female population cannot be adequately established. Additionally, most of the data relating risk factors to coronary heart disease have come from studies of the under 60 age groups and there is really no case for screening - and expecting subsequent benefit-in those over 60: the emphasis should be on the young and middle aged.

While a blood pressure measurement is easy and acceptable to most, a venepuncture is less so; special facilities and improved laboratory services might need to be established in some areas. There are about 12.5 million men aged 15-55 in the United Kingdom. The cost of estimating serum cholesterol concentrations is in the region of $£ 3$, or $£ 37.5$ million for this population.

In order to focus on the top $20 \%$ at highest risk, a repeat estimate would be necessary together with measurement of high density lipoprotein (HDL) cholesterol (at about £3), and this might add $£ 15$ million to the sum. Thus, identification of the top quintile might cost $£ 50$ million or more, but this calculation is for biochemical analyses and not for staff and facilities necessary to do venepunctures. Such expenditure might allow 2.5 million men to receive treatment to lower their very high serum cholesterol. Extrapolating from the Lipid Research Clinic Coronary Primary Prevention Trial results, ${ }^{10}$ coronary heart disease might be postponed or prevented in 
about 70000 men over a seven year period. It is for health economists and politicians to decide if this a worthwhile yield. But there are still other problems.

Screening of the adult male population will identify many with raised cholesterol concentrations and raised blood pressure between the fiftieth and eightieth percentile. What should we tell these people? We know that their risk of coronary heart disease is only slightly increased above that for those in the three deciles below the median, we know that prediction of coronary heart disease is weak and that it cannot be individualised, and we know that mass intervention has so far been singularly disappointing. Even in a health conscious community, I cannot believe that informing these men about their serum cholesterol concentration or blood pressure will be conducive to reassurance so long as the medical profession lacks a strong base for advice or action. Indeed, it is likely to increase anxiety in many. Physicians and cardiologists who support male adult population screening programmes should, therefore, first ask themselves what they will advise when screening identifies an otherwise fit, non-obese, 35 year old man with serum cholesterol concentration or blood pressure in the sixth or eighth deciles. Should he be told? If so, what should he be told about his individual risk and how to reduce it? Should the test be repeated in one or five years time, or should the mildly abnormal result be completely ignored? Each of these questions needs to be identified and answered by policy makers.

\section{Selective screening}

There are problems of comparable difficulty for those who advocate selective screening on the basis of clinical acumen, or case finding. The biggest of theseand there is no information available at present-is the proportion of high risk individuals (above the eightieth percentile) that would be missed by even the most assiduous programmes of clinical screening. Were this to be large, then the strategy of selective screening would probably have to be abandoned in favour of mass screening.

Selective screening may identify currently symptomless individuals with pronounced hypercholesterolaemia; it is less suitable for finding those with severe hypertension, except through hypertensive families. The finding of tendon xanthomata, for which a routine search should be made, points to monogenic familial hypercholesterolaemia, but this only comprises about $15 \%$ of those with severe hypercholesterolaemia with most being polygenic. Identification of a premature corneal arcus or xanthelasma should spread the net a little wider, and it is interesting that opticians do not seem to look for these features routinely. The recent American Heart
Association recommendations ${ }^{14}$ emphasise that family screening can be the key to diagnosis and a big yield might come from the first degree relations of all patients who have developed any clinical features of coronary heart disease under the age of about $50 .{ }^{23} \mathrm{In}$ my experience, it is rare for physicians and cardiologists in hospitals dealing with large numbers of coronary heart disease patients to screen first degree relations routinely for raised serum cholesterol concentrations and blood pressure. This is a matter of improving education since family doctors especially should be able to add considerably to the yield by identifying families with aggregation of vascular disease and screening the apparently healthy relatives.

One advantage of case finding is that the motivation of patients to identify their high risk relatives and of their doctors to intervene will be far higher than that for the general population. It is now up to physicians and cardiologists to join with epidemiologists and demonstrate from population surveys whether or not the majority of those at high risk are identified by selective screening on the basis of case finding.

\section{Improvement of prediction}

Whichever screening strategy is favoured, there will be many who will develop coronary heart disease in whom it was not predicted or predictable. The strengths and weaknesses of the classical risk factors of cigarette smoking, raised cholesterol concentration, and raised blood pressure in predicting coronary heart disease are now well established, and little new in terms of measurements for screening the population for coronary heart disease has become available during the past 10 years.

Sharpening of the methods for identifying those at very high risk is overdue. Perhaps the most obvious and urgent area is prediction of incipient thrombogenesis. Identification of new deoxyribonucleic acid polymorphisms - such as, for example, atherogenic apoprotein gene clusters - which might be applicable to some readily available tissue, like skin, is one hope. Knowledge of the factors that make people intrinsically resistant to coronary heart disease is another, and more studies are needed of the characteristics of families and people who do not get coronary heart disease: it is an area of almost complete ignorance.

One of the greatest priorities, then, for the control of coronary heart disease is to improve both the sensitivity and the specificity with which we are able to predict its development. When this has been achieved the case for screening will be strengthened.

\section{References}

1 Jollans JL. Implementing the NACNE report: an agricultural viewpoint. Lancet 1984; i: 382-3. 
2 The Pooling Project Research Group. Relationship of blood pressure, serum cholesterol, smoking habit, relative weight and ECG abnormalities to incidence of major coronary events. Final report of the pooling project. $\mathcal{f}$ Chronic Dis 1978; 31: 201-306.

3 Dawber TR. The Framingham saudy. Cambridge, Massachusetts: Harvard University Press, 1980: 91-120.

4 Rose G, Hamilton PJS, Keen H, Reid DD, McCartney $P$, Jarrett RJ. Myocardial ischaemia, risk factors and death from coronary heart disease. Lancet 1977; i: 105-9.

5 Multiple Risk Factor Intervention Trial Research Group. Multiple Risk Factor intervention trial: risk factor changes and mortality. $\mathcal{F A M A} 1$ 1982; 248: 1465-78.

6 World Health Organisation. European collaborative group. Multifactorial trial in the prevention of coronary heart disease 3. Incidence and mortality results. Eur Heart f 1983; 4: 141-7.

7 Oliver MF. Should we not forget about mass control of coronary risk factors? Lancet 1983; ii: 37-8.

8 Report of a World Health Organisation expert committee. Prevention of coronary heart disease. WHO Tech Rep Ser 1982; 678: 1-53.

9 Hjermann I, Holme I, Byre KV, Leren P. Effect of diet and smoking intervention on the incidence of coronary heart disease. Lancet 1981; ii: 1303-10.

10 Lipid Research Clinics Coronary Primary Prevention Trial Results. I. Reduction in incidence of coronary heart disease. II. The relationship of reduction in incidence of coronary heart disease to cholesterol lowering. FAMA 1984; 251: 351-74.

11 Pocock SJ. Current issues in the design and interpretation of clinical trials. $\mathrm{Br} \mathrm{Med} \mathrm{F} \mathrm{1985;} \mathrm{290:} \mathrm{39-42.}$

12 Report from the Committee of Principal Investigators. A cooperative trial in the primary prevention of ischaemic heart disease using clofibrate. Br Heart $\mathcal{F}$ 1978; 10: $1069-118$.

13 Turpeinen O, Karvonen MJ, Pekkarinen M, Miettinen
M, Elosuo R, Paavilainen E. Dietary prevention of coronary heart disease: the Finnish Mental Hospital study. Int f Epidemiol 1979; 8: 99-118.

14 Dayton S, Pearce ML, Hashimoto S, Dixon WJ, Tomiyasu U. A controlled clinical trial of a diet high in unsaturated fat in preventing complications of atherosclerosis. Circulation 1969; 40 (suppl 2): 1-63.

15 Oliver MF. Serum cholesterol-the knave of hearts and the joker. Lancet 1981; ii: 1090-5.

16 Committee of Principal Investigators. World Health Organisation cooperative trial on primary prevention of ischaemic heart disease using clofibrate to lower serum cholesterol: the final mortality follow up. Lancet 1984; ii: $600-4$.

17 Peto R. Coronary heart disease prevention: trials of diets to control hyperlipidaemia. In: Miller NE, Lewis B, eds. Lipoproteins atherosclerosis and coronary heart disease. Amsterdam: Elsevier, 1981: 206-8.

18 World Health Organisation/International Society of Hypertension. Mild hypertension liaison committee. Trials of the treatment of mild hypertension. Lancet 1982 ; i: $149-56$.

19 American Heart Association Special Report. Recommendations of the treatment of hyperlipidaemia in adults. Arteriosclerosis 1984; 4: 443A-68A.

20 Multiple Risk Factor Intervention Trial. Control group analyses. Am Heart $\mathcal{F} 1985$ (in press).

21 Heller RF, Chinn S, Tunstall-Pedoe HD, Rose G. How well can we predict coronary heart disease? Findings in the United Kingdom heart disease prevention project. $\mathrm{Br}$ Med F 1984; 288: 1409-11.

22 Oliver MF. Risks of correcting the risks of coronary disease and stroke with drugs. $N$ Engl $f$ Med 1982; 306: 297-8.

23 Oliver MF. The case for intervention in those at high risk for coronary heart disease. In: Ferguson A, ed. Advanced medicine 20. London: Pitman, 1984: 114-24. 